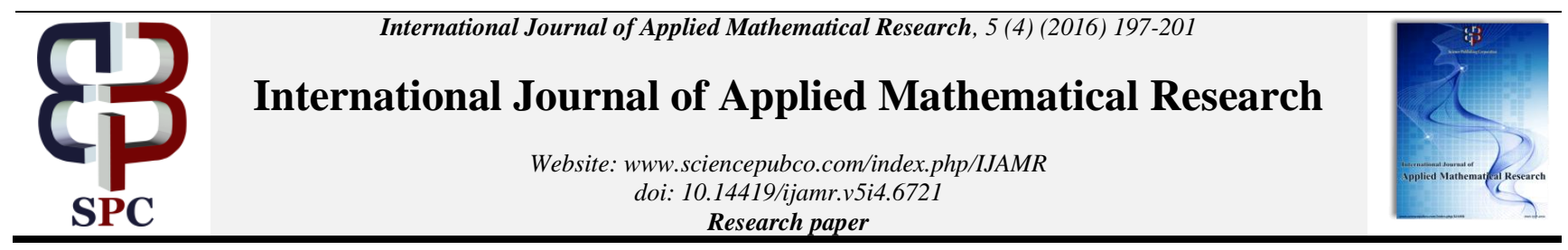

\title{
On common fixed points in generalized Menger spaces
}

\author{
Salwa Salman Abed *, Hadeel Hussein Luaibi \\ Department of Mathematics, College of Education for Pure Sciences Ibn AL - Haitham, University of Baghdad, Iraq \\ *Corresponding author E-mail: salwaalbundi@yahoo.com
}

\begin{abstract}
R. Vasuki [1] proved fixed point theorems for expansive mappings in Menger spaces. R. Gujetiya and et al [2] presented an extension of the main result of Vasuki, for four expansive mappings in Menger space. In this article, an important lemma is given to prove that the iteration sequence is Cauchy under suitable condition in Menger probabilistic G-metric space (shortly, MPGM-space). And then, used to obtain three common fixed point theorems for expansive type mappings.
\end{abstract}

Keywords: Fixed Points; Weakly Compatible; Semi-Compatible; Menger Spaces.

\section{Introduction}

The metric fixed point theory plays an important role to detection many applications in other mathematical branches such as differential equation, operation research, mathematical economics fractals, chaos. Different generalizations of the usual notion of a metric space were defined by several mathematicians such as in Dolthinov [3], Czerwik [4], Branceciri [5], Naidu [6] and Huang and Zhang in [7],. Also G-metric space is of these generalizations which introduced by Mustafa and Sims [8]. For more details and results in G- metric space about fixed points, common fixed points and coincidence points or coupled (fixed point, common fixed points and coincidence points) for mappings satisfying various contractive conditions in co G-metric spaces , ordered G-metric spaces and G-cone metric spaces, such as see [9], [10], [11], [12], [13] and [14].

On the other hand, as a generalization of metric space, K. Menger [15], introduced the concept of a probabilistic metric space (briefly, PM-space) where the notion of distance is considered to be statistical or probabilistic. The definition of PM-space corresponds to conjuncture when we do not know exactly the distance between two points, but we know probabilities of possible values of this distance. The fundamental importance of PM- theory in probabilistic functional analysis due to its extensive applications in random differential as well as random integral equations, for example, the work due to chang and et al [16]. In the field of fixed point, Sehgal [17] presented an active study about the contraction mapping in PM-spaces. Segal and Bharucha-Reid [18] studied Banach's contraction theorem in complete Menger space. See also [18], [19], [9] and [20]. In [21], Hicks observed that interesting fixed point theorems for contraction mappings on a Menger spaces endowed with a triangular $\mathrm{t}$ - norm. Recently, the M. Janfada, A. Janfada and Z. Mollace [22] introduced the structure of probabilistic G-metric spaces and Menger probabilistic G-metric spaces and showed some basic properties about these spaces and then proved some fixed point theorems in it. Abed and Luaibi [23] define a GPM-space and use it to show that proved some fixed point theorem and common fixed point results for convers commuting mappings and weakly compatible mappings in G- Menger space by using implicit conditions.
This paper is included some results about unique common fixed points in probabilistic G-Menger metric space in two various situations.

\section{Preliminaries}

We be gain with same basic definitions and facts.

Definition 1.1: [8] Let $X$ be a nonempty set and $G: X \times X \times X \rightarrow$ $[0,+\infty)$ be a function for all $x, y, z$, a in $X$ satisfying the following conditions:

1) $\mathrm{G}(\mathrm{x}, \mathrm{y}, \mathrm{z})=0 \Leftrightarrow \mathrm{x}=\mathrm{y}=\mathrm{z}$

2) $0<\mathrm{G}(\mathrm{x}, \mathrm{x}, \mathrm{y})$ with $\mathrm{x} \neq \mathrm{y}$

3) $G(x, x, y) \leq G(x, y, z)$ with $y \neq z$

4) $G(x, y, z)=G(p(x, z, y)), p(x, y, z)$ is a permutation of $x, y, z$

5) $\quad G(x, y, z) \leq G(x, a, a)+G(a, y, z)$.

Then the ordered pair $(X, G)$ is called a generalized metric space or G- metric space.

Definition 1.2: [8] Let $(X, G)$ be a $G$ - metric space. The sequence $\left\{x_{n}\right\}$ is called.

1) A G-Cauchy if, $\forall \varepsilon>0$, there is $\mathrm{k} \in \mathrm{N}$ such that for all positive integers $n, m, 1 \geq k, G\left(x_{n}, x_{m}, x_{l}\right)<\varepsilon$.

2) A G-convergent to $x \in X$ if, $\forall \varepsilon>0$, there is $k \in N$ such that for all $\mathrm{n}, \mathrm{m} \geq \mathrm{k}, \mathrm{G}\left(\mathrm{x}, \mathrm{x}_{\mathrm{n}}, \mathrm{x}_{\mathrm{m}}\right)<\varepsilon$.

Also, $(X, G)$ is said to be complete G-metric space if every GCauchy sequence in $\mathrm{X}$ is $\mathrm{G}$ - convergent in $\mathrm{G}$.

Definition 1.3: $[24]$ the mapping $\Delta:[0,1] \times[0,1] \rightarrow[0,1]$, is called a continuous $t$ - norm if $\Delta$ satisfies the following conditions.

a) $\Delta(\mathrm{r}, 1)=\mathrm{r}$ for all $\mathrm{r}$ in $[0,1]$;

b) $\Delta(\mathrm{r}, \mathrm{s})=\Delta(\mathrm{s}, \mathrm{r})$, for every $\mathrm{r}, \mathrm{s} \in[0,1]$;

c) $\quad \Delta(\mathrm{a}, \mathrm{c}) \geq \Delta(\mathrm{b}, \mathrm{d})$, whenever $\mathrm{a} \geq \mathrm{b}$ and $\mathrm{c} \geq \mathrm{d}$, for each $\mathrm{a}, \mathrm{b}, \mathrm{c}$, $\mathrm{d}$ in $[0,1]$;

d) $\Delta$ is continuous;

e) (e) $\Delta(\mathrm{r}, \Delta(\mathrm{s}, \mathrm{c}))=\Delta(\Delta(\mathrm{r}, \mathrm{s}), \mathrm{c}),(\mathrm{r}, \mathrm{s}, \mathrm{c} \in[0,1])$.

Example 1.4: [24] The following are the four basic t-norms.

1) The minimum: $\Delta_{\mathrm{M}}(\mathrm{r}, \mathrm{s})=\min \{\mathrm{r}, \mathrm{s}\}$. 
2) The product: $\Delta_{\mathrm{P}}(\mathrm{r}, \mathrm{s})=\mathrm{r}$ s.

3) The Lukasiewicz: $\Delta_{\mathrm{L}}(\mathrm{r}, \mathrm{s})=\max \{\mathrm{r}+\mathrm{s}-1,0\}$.

4) The weakest:

$\Delta \mathrm{D}(\mathrm{r}, \mathrm{s})=\left\{\begin{array}{c}\min \{\mathrm{r}, \mathrm{s}\} \text { if } \max \{\mathrm{r}, \mathrm{s}\}=1, \\ 0 \text { otherwise. }\end{array}\right.$

In respect of the above mentioned t-norms, we have the following ordering:

$$
\Delta_{\mathrm{D}}<\Delta_{\mathrm{L}}<\Delta \mathrm{P}<\Delta_{\mathrm{M}}
$$

Here, we give anther version of the definition of probabilistic Gmetric space. Our idea depend on the definition in usual G-metric which different from the definition in [22]:

Definition 1.5: A Menger Probabilistic G- Metric space (briefly, Menger PGM-space) is a triple $(X, G, \Delta)$, where $X$ is a non-empty set, $\Delta$ is a continuous $\mathrm{t}$ - norm, and $\mathrm{G}$ is a mapping from $\mathrm{X} \times \mathrm{X} \times$ $X$ into $L$ such that, if $G_{x, y, z}$ denotes the value of $G$ at the triple ( $x$, $\mathrm{y}, \mathrm{z}$ ), the following conditions hold: for all $\mathrm{x}, \mathrm{y}, \mathrm{z}$ in $\mathrm{X}$,

1) $G_{x, y, z}(t)<1$ for all $t>0$ if and only if $x \neq y$;

2) $G_{x, y, z}(t)=1$ for all $t>0$ if and only if $x=y=z$;

3) $G_{x, x, y}(t) \geq G_{x, y, z}(t)$

4) $\mathrm{G}_{\mathrm{x}, \mathrm{y}, \mathrm{z}}(\mathrm{t})=\mathrm{G}_{\mathrm{y}, \mathrm{z}, \mathrm{x}}(\mathrm{t})=\mathrm{G}_{\mathrm{z}, \mathrm{x}, \mathrm{y}}(\mathrm{t}) \ldots$,

5) $G_{x, y, z}(t+s) \geq \Delta\left(G_{x, a, a}(t), G_{a, y, z}(s)\right)$ for all $x, y, z, a \in X$ and $\mathrm{t}, \mathrm{s} \geq 0$,

6) If $G_{x, a, a}(t)=G_{a, y, z}(s)=1$ then $G_{x, y, z}(t+s)=1$.

Note that, when $(\mathrm{X}, \mathrm{G}, \Delta)$ satisfies the conditions $1,2,3,4$ and 6 in definition (1.5) then it is called Probabilistic G- Metric space (briefly, PGM-space)

Definition 1.6: [25] Let $(X, G, \Delta)$ be a Menger PGM-space.

1) A sequence $\left\{x_{n}\right\}$ in $X$ is said to be $P G$ - convergent to $x$ in $X$ if, for every $\varepsilon>0$ and $\lambda>0$, there exists positive integer $\mathrm{M}(\varepsilon, \lambda)$ such that.

$$
\mathrm{G}_{\mathrm{x}, \mathrm{x}_{\mathrm{n}}, \mathrm{x}_{\mathrm{m}}}(\varepsilon)>1-\lambda \text { whenever } \mathrm{m}, \mathrm{n} \geq \mathrm{M}(\varepsilon, \lambda) .
$$

2) A sequence $\left\{x_{n}\right\}$ in $X$ is called PG- Cauchy sequence if, for every $\varepsilon>0$ and $\lambda>0$, there exists positive integer $\mathrm{M}(\varepsilon, \lambda)$ such that

$$
\mathrm{G}_{\mathrm{x}_{\mathrm{n}}, \mathrm{x}_{\mathrm{m}}, \mathrm{x}_{\mathrm{l}}}(\varepsilon)>1-\lambda \text { whenever } \mathrm{n}, \mathrm{m}, \mathrm{l} \geq \mathrm{M}(\varepsilon, \lambda)
$$

3) A Menger PM-space $(X, G, \Delta)$ is said to be complete if and only if every PG- Cauchy sequence in $\mathrm{X}$ is PG- convergent to a point in $\mathrm{X}$.

Definition 1.7: [25] A pair of maps $T$ and $S$ is called weakly compatible pair if they commute at coincidence points i.e., $T x=S x$ implies $T S x=S T x$.

Definition 1.8: Self- mappings $S$ and $T$ of a Menger PGM-space $(X, G, \Delta)$ are said to be semi-compatible if $G_{S T x_{n}, T u, T u}(t) \rightarrow 1$ for all $t>0$, whenever $\left\{x_{n}\right\}$ is a sequence in $X$ such that $S x_{n}, T x_{n} \rightarrow$ for some $u$ in $X$, as $n \rightarrow \infty$.

It follows that the pair $(\mathrm{S}, \mathrm{T})$ is semi-compatible and Sy =Ty imply STy $=$ TSy by taking $\left\{x_{n}\right\}=y$ and $u=S y=T y$.

\section{Main results}

Firstly, the following lemmas are needed:

Lemma 2.1: Let $\left(X, G, \Delta_{M}\right)$ be a Menger PGM-space and $\left\{x_{n}\right\}$ be a sequence in $X$. If there exists a positive number, $0<q<1$ and $t>$ $O$ and $\Delta_{M}=\min$

Such that

$$
G_{x_{n}, x_{n+1}, x_{m}}(q t) \geq G_{x_{n-1}, x_{n}, x_{m-1}}(t)
$$

For all $\mathrm{m} \geq \mathrm{n}+1, \mathrm{n}=1,2,3 \ldots$

Then $\left\{x_{n}\right\}$ is a PG- Cauchy sequence in $X$.

Proof:

It follows from (2.1),

$G_{x_{n}, x_{n-1}, x_{m}}((1-q) \varepsilon / 2 q) \geq G_{x_{n-1}, x_{n-2}, x_{m-1}}\left((1-q) \varepsilon / 2 q^{2}\right)$

$$
\geq \ldots \geq G_{x_{2}, x_{1}, x_{m-n+2}}\left((1-q) \varepsilon / 2 q^{n-1}\right)
$$

Since $0<\mathrm{q}<1$, so for $\varepsilon>0$ and $\lambda>0$, there exists a positive integer $\mathrm{K}=\mathrm{M}(\varepsilon, \lambda)$ such that

$\mathrm{G}_{\mathrm{x}_{\mathrm{n}}, \mathrm{x}_{\mathrm{n}-1}, \mathrm{x}_{\mathrm{m}}}((1-\mathrm{q}) \varepsilon / 2 \mathrm{q})>1-\lambda$, for all $\mathrm{n} \geq \mathrm{K}$

It is sufficient to prove that for any positive integer $p$,

$$
\mathrm{G}_{\mathrm{x}_{\mathrm{n}}, \mathrm{x}_{\mathrm{n}+\mathrm{p}}, \mathrm{x}_{\mathrm{m}}}(\varepsilon) \geq 1-\lambda, \mathrm{n} \geq \mathrm{K}
$$

For $\mathrm{p}=1,(2.3)$ holds. Suppose that (2.1) holds for $1<\mathrm{p} \leq \mathrm{k}$, then

$$
\begin{aligned}
\mathrm{G}_{\mathrm{x}_{\mathrm{n}}, \mathrm{x}_{\mathrm{n}+\mathrm{k}+1}, \mathrm{x}_{\mathrm{m}}}(\varepsilon) & \geq \mathrm{G}_{\mathrm{x}_{\mathrm{n}-1}, \mathrm{x}_{\mathrm{n}+\mathrm{k}}, \mathrm{x}_{\mathrm{m}-1}}(\varepsilon / 2) \\
& \geq \min \left\{\mathrm{G}_{\mathrm{x}_{\mathrm{n}-1}, \mathrm{x}_{\mathrm{n}}, \mathrm{x}_{\mathrm{n}}}((1-\mathrm{q}) \varepsilon / 2 \mathrm{q}), \mathrm{G}_{\mathrm{x}_{\mathrm{n}}, \mathrm{x}_{\mathrm{n}+\mathrm{k}}, \mathrm{x}_{\mathrm{m}-1}}(\varepsilon)\right\} \\
& =\min \left\{\mathrm{G}_{\mathrm{x}_{\mathrm{n}}, \mathrm{x}_{\mathrm{n}}, \mathrm{x}_{\mathrm{n}-1}}((1-\mathrm{q}) \varepsilon / 2 \mathrm{q}), \mathrm{G}_{\mathrm{x}_{\mathrm{n}}, \mathrm{x}_{\mathrm{n}+\mathrm{k}}, \mathrm{x}_{\mathrm{m}-1}}(\varepsilon)\right\} \\
& \geq \min \left\{\mathrm{G}_{\mathrm{x}_{\mathrm{n}}, \mathrm{x}_{\mathrm{n}-1}, \mathrm{x}_{\mathrm{m}}}((1-\mathrm{q}) \varepsilon / 2 \mathrm{q}), \mathrm{G}_{\mathrm{x}_{\mathrm{n}}, \mathrm{x}_{\mathrm{n}+\mathrm{k}}, \mathrm{x}_{\mathrm{m}-1}}(\varepsilon)\right\}
\end{aligned}
$$

[By (3) from definition (0.6.1)]

$>\min \{1-\lambda, 1-\lambda\}=1-\lambda, \mathrm{n} \geq \mathrm{K}$.

Hence (2. 3) holds for $\mathrm{p}=\mathrm{k}+1$. Therefore, $\left\{\mathrm{x}_{\mathrm{n}}\right\}$ is a PG-Cauchy sequence.

Lemma 2.3: Let $(X, G, \Delta)$ be a Menger PGM - space. If there exists $k \in(0,1)$ such that for $x, y, z \in X, G_{x, y, z}(k t)=1$ then $x=y$ $=z$.

From the definition of Menger PGM - space, the poof will be obviously.

The first result:

Theorem 2.4: Let $T, S: X \rightarrow X$ be a weakly compatible mappings on a Menger PGM - space $(X, G, \Delta M)$ with

$$
\mathrm{G}_{\mathrm{Sx}, \mathrm{Sy}, \mathrm{Sz}}(\mathrm{k} \mathrm{t}) \leq \mathrm{G}_{\mathrm{Tx}, \mathrm{Ty}, \mathrm{Tz}}(\mathrm{t})
$$

For all $\mathrm{x}, \mathrm{y}, \mathrm{z}$ in $\mathrm{X}, \mathrm{k}>1$ and $\mathrm{t}>0$. Then $\mathrm{T}$ and $\mathrm{S}$ have a unique common fixed point, whenever $\mathrm{T}(\mathrm{X}) \subseteq \mathrm{S}(\mathrm{X})$ and $\mathrm{T}(\mathrm{X})$ or $\mathrm{S}(\mathrm{X})$ is complete.

Proof:

Let $\mathrm{x}_{0} \in \mathrm{X}$ be an arbitrary point in $\mathrm{X}$ such that $\mathrm{y}_{0}=\mathrm{Sx}_{0}$. Since $\mathrm{T}(\mathrm{X}) \subseteq \mathrm{S}(\mathrm{X})$, so we can choose a point $\mathrm{x}_{1}$ in $\mathrm{X}$ such that $\mathrm{y}_{1}=\mathrm{Tx}_{0}$ $=\mathrm{Sx}_{1}$. 
In general, choose $\mathrm{x}_{\mathrm{n}+1}$ such that $\mathrm{y}_{\mathrm{n}+1}=\mathrm{Tx}_{\mathrm{n}}=\mathrm{Sx}_{\mathrm{n}+1}, \mathrm{n}=0,1,2$ ...

Then from (2.4)

$$
\begin{aligned}
G_{y_{n-1}, y_{n}, y_{m-1}}(k t) & =G_{S_{x_{n-1}}, S_{x_{n}}}, S_{x_{m-1}}(k t) \\
\leq & G_{T_{x_{n-1}}, T_{x_{n}}}, T_{x_{m-1}}(t) \\
= & G_{y_{n}, y_{n+1}, y_{m}}(t) .
\end{aligned}
$$

Then, $G_{y_{n}, y_{n+1}, y_{m}}(q t) \geq G_{y_{n-1}, y_{n}, y_{m-1}}(t)$, where $q=1 / k$

By Lemma (2.1), we get $\left\{y_{n}\right\}$ is a PG-Cauchy and hence convergent. If we denote the limit by $u$, then

$\lim _{\mathrm{n} \rightarrow \infty} \operatorname{Tx}_{\mathrm{n}}=\lim _{\mathrm{n} \rightarrow \infty} \mathrm{Sx}_{\mathrm{n}}=\lim _{\mathrm{n} \rightarrow \infty} \mathrm{y}_{\mathrm{n}}=\mathrm{u}$.

Since $S(X)$ is complete, there exists a point $p \in X$ such that $S p=u$

Now from (2.4)

$\mathrm{G}_{\mathrm{Sp}, \mathrm{S}_{\mathrm{x}_{\mathrm{n}}}, \mathrm{S}_{\mathrm{x}_{\mathrm{n}}}}(\mathrm{kt}) \leq \mathrm{G}_{\mathrm{Tp}, \mathrm{T}_{\mathrm{x}_{\mathrm{n}}}, \mathrm{T}_{\mathrm{x}_{\mathrm{n}}}}(\mathrm{t})$

Taking limit as $\mathrm{n} \rightarrow \infty$ we have

$\mathrm{G}_{\mathrm{Sp}, \mathrm{u}, \mathrm{u}}(\mathrm{k} \mathrm{t}) \leq \mathrm{G}_{\mathrm{Tp}, \mathrm{u}, \mathrm{u}}(\mathrm{t})$, which implies that $\mathrm{Tp}=\mathrm{u}$. Therefore, $\mathrm{Sp}$ $=\mathrm{Tp}=\mathrm{u}$.

Since $\mathrm{S}$ and $\mathrm{T}$ are weakly compatible, therefore, $\mathrm{ST} p=\mathrm{TSp}$ i.e., $\mathrm{Su}=\mathrm{Tu}$.

Now we will prove that $\mathrm{u}$ is a fixed point of $\mathrm{S}$ and $\mathrm{T}$. From (2.4)

$$
\mathrm{G}_{\mathrm{Su}, \mathrm{S}_{\mathrm{x}_{\mathrm{n}}}, \mathrm{S}_{\mathrm{x}_{\mathrm{n}}}}(\mathrm{k} \mathrm{t}) \leq \mathrm{G}_{\mathrm{Tu}, \mathrm{T}_{\mathrm{x}_{\mathrm{n}}}, \mathrm{T}_{\mathrm{x}_{\mathrm{n}}}}(\mathrm{t})
$$

Taking limit as $\mathrm{n} \rightarrow \infty$, we have

$$
\mathrm{G}_{\mathrm{Su}, \mathrm{u}, \mathrm{u}}(\mathrm{k} \mathrm{t}) \leq \mathrm{G}_{\mathrm{Tu}, \mathrm{u}, \mathrm{u}}(\mathrm{t})
$$

Which implies that $\mathrm{Tu}=\mathrm{u}$. Hence $\mathrm{Tu}=\mathrm{Su}=\mathrm{u}$.

Now, suppose that $\mathrm{u} \neq \mathrm{w}$ is also another common fixed point of $\mathrm{S}$ and $\mathrm{T}$.

Then from (2.4)

$\mathrm{G}_{\mathrm{Su}, \mathrm{Sw}, \mathrm{Sw}}(\mathrm{kt}) \leq \mathrm{G}_{\mathrm{Tu}, \mathrm{Tw}, \mathrm{Tw}}(\mathrm{t})$, this implies $\mathrm{u}=\mathrm{w}$.

Proposition 2.5: If ( $\mathrm{S}, \mathrm{T})$ is a semi-compatible pair of self- mappings in a Menger PGM - space $(X, G, \Delta M)$ and $T$ is continuous then $(\mathrm{S}, \mathrm{T})$ is compatible.

Proof:

Consider a sequence $\left\{x_{n}\right\}$ in $X$ such that $\left\{\mathrm{Sx}_{\mathrm{n}}\right\} \rightarrow \mathrm{u}$ and $\left\{\mathrm{Tx}_{\mathrm{n}}\right\}$ $\rightarrow \mathrm{u}$. As $\mathrm{T}$ is continuous we get $\mathrm{TS} \mathrm{x}_{\mathrm{n}} \rightarrow \mathrm{Tu}$. By semicompatibility of (S, T), we have $\mathrm{G}_{\mathrm{STx}_{\mathrm{n}}, \mathrm{Tu}, \mathrm{Tu}}(\varepsilon) \rightarrow 1$ for all $\varepsilon>0$, i.e. for $\varepsilon>0$ and $\lambda>0$, there is an integer $M(\varepsilon, \lambda)$ such that , $\mathrm{G}_{\mathrm{STx}_{\mathrm{n}}, \mathrm{Tu}, \mathrm{Tu}}(\varepsilon / 2) \rightarrow 1-\lambda$ and, $\mathrm{G}_{\mathrm{TSx}_{\mathrm{n}}, \mathrm{Tu}, \mathrm{Tu}}(\varepsilon / 2) \rightarrow 1-\lambda$ for all $\mathrm{n} \geq$ $\mathrm{M}(\varepsilon, \lambda)$.

Now $\mathrm{G}_{\mathrm{STx}_{\mathrm{n}}, \mathrm{TSu}, \mathrm{TSu}}(\varepsilon)>\Delta\left\{\mathrm{G}_{\mathrm{TSx}_{\mathrm{n}}, \mathrm{Tu}, \mathrm{Tu}}(\varepsilon / 2), \mathrm{G}_{\mathrm{Tu}_{\mathrm{TSx}}, \mathrm{TSx}_{\mathrm{n}}}(\varepsilon / 2)\right\}$

$$
\begin{aligned}
& >\Delta\left\{\mathrm{G}_{\mathrm{TSx}_{\mathrm{n}}, \mathrm{Tu}, \mathrm{Tu}}(\varepsilon / 2), \mathrm{G}_{\mathrm{TSx}_{\mathrm{n}}, \mathrm{Tu}, \mathrm{Tu}}(\varepsilon / 2)\right\} \\
& >(1-\lambda, 1-\lambda)>1-\lambda .
\end{aligned}
$$

we get $\mathrm{G}_{\mathrm{STx}_{\mathrm{n}}, \mathrm{TSu}, \mathrm{TSu}}(\varepsilon) \rightarrow 1$ for all $\varepsilon>0$.

Hence, the pair $(S, T)$ is compatible.
Theorem 2.6: Let (X, G, $\Delta_{\mathrm{M}}$ be a complete Menger PGM - space with a probabilistic G- metric is symmetric and the mappings $\mathrm{A}$, $\mathrm{B}, \mathrm{S}$ and $\mathrm{T}: \mathrm{X} \rightarrow \mathrm{X}$ mappings with

$\mathrm{G}_{\mathrm{Ax}, \mathrm{By}, \mathrm{By}}(\mathrm{k}) \leq \min \left\{\mathrm{G}_{\mathrm{Sx}, \mathrm{Sx}, \mathrm{Ax}}(\mathrm{k} / \mathrm{d}), \mathrm{G}_{\mathrm{Ty}, \mathrm{Ty}, \mathrm{By}}(\mathrm{k} / \mathrm{e}), \mathrm{G}_{\mathrm{Sx}, \mathrm{Ty}, \mathrm{Ty}}(\mathrm{k}\right.$ /f) $\}$

For some $\mathrm{k} \in(0,1)$ and for all $\mathrm{x}, \mathrm{y} \in \mathrm{X}$ with $\mathrm{x} \neq \mathrm{y}$ where $\beta=$ $\min \{\mathrm{d}, \mathrm{e}, \mathrm{f}\}>1$. Then $\mathrm{A}, \mathrm{B}, \mathrm{S}$ and $\mathrm{T}$ have a unique common fixed point in $\mathrm{X}$, if: (i) $(\mathrm{A}, \mathrm{S})$ is semi-compatible and $(\mathrm{B}, \mathrm{T})$ is weak compatible; (ii) $\mathrm{T}(\mathrm{X}) \subseteq \mathrm{A}(\mathrm{X})$ and $\mathrm{S}(\mathrm{X}) \subseteq \mathrm{B}(\mathrm{X})$; and (iii) (i) either A or $\mathrm{S}$ is continuous.

Proof:

Let $\mathrm{x}_{0} \in \mathrm{X}$, by condition (i) there exist $\mathrm{x}_{1}, \mathrm{x}_{2} \in \mathrm{X}$ such that

$\mathrm{Tx}_{0}=\mathrm{Ax}_{1}=\mathrm{y}_{0} ; \mathrm{Sx}_{1}=\mathrm{Bx}_{2}=\mathrm{y}_{1}$

Inductively, we can construct sequences $\left\{x_{n}\right\}$ and $\left\{y_{n}\right\}$ in $X$ such that

$$
\mathrm{Tx}_{2 \mathrm{n}}=\mathrm{Ax}_{2 \mathrm{n}+1}=\mathrm{y}_{2 \mathrm{n}} ; \mathrm{Sx}_{2 \mathrm{n}+1}=\mathrm{Bx}_{2 \mathrm{n}+2}=\mathrm{y}_{2 \mathrm{n}+1}
$$

Now,

$$
\begin{aligned}
\mathrm{G}_{\mathrm{y}_{0}, \mathrm{y}_{1}, \mathrm{y}_{1}}(\mathrm{k})=\mathrm{G}_{\mathrm{Ax}_{1}, \mathrm{Bx}_{2}, \mathrm{Bx}_{2}}(\mathrm{k}) \\
\quad \leq \min \left\{\mathrm{G}_{\mathrm{Sx}_{1}, \mathrm{Sx}_{1}, \mathrm{Ax}_{1}}(\mathrm{k} / \mathrm{d}), \mathrm{G}_{\mathrm{Tx}_{2}, \mathrm{Tx}_{2}, \mathrm{Bx}_{2}}(\mathrm{k} / \mathrm{e}), \mathrm{G}_{\mathrm{Sx}_{1}, \mathrm{Tx}_{2}, \mathrm{Tx}_{2}}(\mathrm{k} / \mathrm{f})\right\} \\
\quad \leq \min \left\{\mathrm{G}_{\mathrm{y}_{1}, \mathrm{y}_{1}, \mathrm{y}_{0}}(\mathrm{k} / \mathrm{d}), \mathrm{G}_{\mathrm{y}_{2}, \mathrm{y}_{2}, \mathrm{y}_{1}}(\mathrm{k} / \mathrm{e}), \mathrm{G}_{\mathrm{y}_{1}, \mathrm{y}_{2}, \mathrm{y}_{2}}(\mathrm{k} / \mathrm{f})\right\} \\
\quad \leq \min \left\{\mathrm{G}_{\mathrm{y}_{0}, \mathrm{y}_{1}, \mathrm{y}_{1}}(\mathrm{k} / \beta), \mathrm{G}_{\mathrm{y}_{1}, \mathrm{y}_{2}, \mathrm{y}_{2}}(\mathrm{k} / \beta), \mathrm{G}_{\mathrm{y}_{1}, \mathrm{y}_{2}, \mathrm{y}_{2}}(\mathrm{k} / \beta)\right\},
\end{aligned}
$$

Where $\beta=\min \{d, e, f\}$

$$
\begin{aligned}
& \leq \min \left\{\mathrm{G}_{\mathrm{y}_{0}, \mathrm{y}_{1}, \mathrm{y}_{1}}(\mathrm{k} / \beta), \mathrm{G}_{\mathrm{y}_{1}, \mathrm{y}_{2}, \mathrm{y}_{2}}(\mathrm{k} / \beta)\right\}, \\
& \leq \min \left\{\mathrm{G}_{\mathrm{y}_{1}, \mathrm{y}_{2}, \mathrm{y}_{2}}(\mathrm{k} / \beta)\right\}=\mathrm{G}_{\mathrm{y}_{1}, \mathrm{y}_{2}, \mathrm{y}_{2}}(\mathrm{k} / \beta)
\end{aligned}
$$

i.e. $G_{y_{0}, y_{1}, y_{1}}(k) \leq G_{y_{1}, y_{2}, y_{2}}(k / \beta)$. Similarly, $G_{y_{1}, y_{2}, y_{2}}(k) \leq$ $\mathrm{G}_{\mathrm{y}_{2}, \mathrm{y}_{3}, \mathrm{y}_{3}}(\mathrm{k} / \beta)$

Now, again

$$
\begin{aligned}
& \mathrm{G}_{\mathrm{y}_{2 \mathrm{n}}, \mathrm{y}_{2 \mathrm{n}+1}, \mathrm{y}_{2 \mathrm{n}+1}}(\mathrm{k})=\mathrm{G}_{\mathrm{Ax}_{2 \mathrm{n}+1}, \mathrm{Bx}_{2 \mathrm{n}+2}, \mathrm{Bx}_{2 \mathrm{n}+2}}(\mathrm{k}) \\
& \quad \leq \min \left\{\mathrm{G}_{\mathrm{Sx}_{2 \mathrm{n}+1}, \mathrm{Sx}_{2 \mathrm{n}+1}, \mathrm{Ax}_{2 \mathrm{n}+1}}(\mathrm{k} / \mathrm{d}), \mathrm{G}_{\mathrm{Tx}_{2 \mathrm{n}+2}, \mathrm{Tx}_{2 \mathrm{n}+2}, \mathrm{Bx}_{2 \mathrm{n}+2}}(\mathrm{k} / \mathrm{e}),\right.
\end{aligned}
$$

$\left.\mathrm{G}_{\mathrm{Sx}_{2 \mathrm{n}+1}, \mathrm{Tx}_{2 \mathrm{n}+2}, \mathrm{Tx}_{2 \mathrm{n}+2}}(\mathrm{k} / \mathrm{f})\right\}$

$\leq \min \left\{G_{y_{2 n+1}, y_{2 n+1}, y_{2 n}}(k / d), G_{y_{2 n+2}, y_{2 n+2}, y_{2 n+1}}(k / e\right.$

), $\left.\mathrm{G}_{\mathrm{y}_{2 \mathrm{n}+1}, \mathrm{y}_{2 \mathrm{n}+2}, \mathrm{y}_{2 \mathrm{n}+2}}(\mathrm{k} / \mathrm{f})\right\}$

$\leq \min \left\{G_{y_{2 n}, y_{2 n+1}, y_{2 n+1}}(k / \beta), G_{y_{2 n+1}, y_{2 n+2}, y_{2 n+2}}(k / \beta)\right.$,

$\left.\mathrm{G}_{\mathrm{y}_{2 \mathrm{n}+1}, \mathrm{y}_{2 \mathrm{n}+2}, \mathrm{y}_{2 \mathrm{n}+2}}(\mathrm{k} / \beta)\right\}$,

Where $\beta=\min \{d, e, f\}$

$\leq \min \left\{G_{y_{2 n}, y_{2 n+1}, y_{2 n+1}}(k / \beta), G_{y_{2 n+1}, y_{2 n+2}, y_{2 n+2}}(k / \beta)\right\}$,

$\leq \min \left\{G_{y_{2 n+1}, y_{2 n+2}, y_{2 n+2}}(k / \beta)\right\}=G_{y_{2 n+1}, y_{2 n+2}, y_{2 n+2}}(k / \beta)$

i.e. $G_{y_{2 n}, y_{2 n+1}, y_{2 n+1}}(k) \leq G_{y_{2 n+1}, y_{2 n+2}, y_{2 n+2}}(k / \beta), \beta>1$

Now again

$\mathrm{G}_{\mathrm{y}_{2 n+1}, \mathrm{y}_{2 \mathrm{n}+2}, \mathrm{y}_{2 \mathrm{n}+2}}(\mathrm{k})=\mathrm{G}_{\mathrm{y}_{2 n+2}, \mathrm{y}_{2 \mathrm{n}+1}, \mathrm{y}_{2 \mathrm{n}+1}}(\mathrm{k})$

$$
=\mathrm{G}_{\mathrm{Ax}_{2 \mathrm{n}+3}, \mathrm{Bx}_{2 \mathrm{n}+2}, \mathrm{Bx}_{2 \mathrm{n}+2}}(\mathrm{k})
$$




$$
\begin{aligned}
& \leq \min \left\{G_{S_{x_{2 n+3},}, S_{2 n+3}, A x_{2 n+3}}(k / d), G_{T_{x_{2 n}+2}, T_{2 n+2}, B_{2 n+2}}(k / e),\right. \\
& \leq \min \left\{G_{y_{2 n+3}, y_{2 n+3}, y_{2 n+2}}(k / d), G_{y_{2 n+2}, y_{2 n+2}, y_{2 n+1}}(k / e),\right. \\
& \left.G_{S_{2 n+3}, T_{2 n+2}, T_{x_{2 n+2}}}(k / f)\right\} \\
& \leq \min \left\{G_{y_{2 n+3}, y_{2 n+2}, y_{2 n+2}}(k / f)\right\} \\
& \left.G_{y_{2 n+2}, y_{2 n+3}, y_{2 n+3}}(k / \beta)\right\},
\end{aligned}
$$

Where $\beta=\min \{\mathrm{d}, \mathrm{e}, \mathrm{f}\}$

Hence, $G_{y_{2 n+1}, y_{2 n+2}, y_{2 n+2}}(k) \leq G_{y_{2 n+2}, y_{2 n+3}, y_{2 n+3}}(k / \beta)$, as $\beta>1$

Hence for all $n$, we have $G_{y_{2 n}, y_{2 n+1}, y_{2 n+1}}(k) \leq G_{y_{2 n+1}, y_{2 n+2}, y_{2 n+2}}(k /$ $\beta)$, as $\beta>1$

Now,

$$
\begin{aligned}
& G_{y_{2 n}, y_{2 n+1}, y_{2 n+1}}(k) \leq G_{y_{2 n+1}, y_{2 n+2}, y_{2 n+2}}(v k) \text { as } v=1 / \beta, \beta>1 \\
& G_{y_{2 n+1}, y_{2 n+2}, y_{2 n+2}}(v k) \geq G_{y_{2 n}, y_{2 n+1}, y_{2 n+1}}(k)
\end{aligned}
$$

By lemma (3.1), we get $\left\{y_{n}\right\}$ is a PG-Cauchy sequence in X. Thus there exist some point $\mathrm{z} \in \mathrm{X}$ to which $\left\{\mathrm{y}_{\mathrm{n}}\right\}$ converges. Now its subsequences

$$
\left\{\mathrm{Ax}_{2 \mathrm{n}+1}\right\} \rightarrow \mathrm{z},\left\{\mathrm{Bx}_{2 \mathrm{n}+2}\right\} \rightarrow \mathrm{z},\left\{\mathrm{Sx}_{2 \mathrm{n}+1}\right\} \rightarrow \mathrm{z},\left\{\mathrm{Tx}_{2 \mathrm{n}+2}\right\} \rightarrow \mathrm{z}
$$

Case I: $\mathrm{S}$ is continuous.

In this case, we have

$\mathrm{SAx}_{2 \mathrm{n}+1} \rightarrow \mathrm{Sz}$ and $\mathrm{S}^{2} \mathrm{x}_{2 \mathrm{n}+1} \rightarrow \mathrm{Sz}$

The semi-compatibility of $(A, S)$, gives $\mathrm{SAx}_{2 \mathrm{n}+1} \rightarrow \mathrm{Sz}$.

Step 1. By putting $\mathrm{x}=\mathrm{Sx}_{2 \mathrm{n}+1}, \mathrm{y}=\mathrm{x}_{2 \mathrm{n}+2}$ in (iv), we have

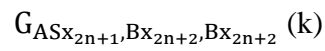

$$
\begin{aligned}
& \leq \min \left\{G_{S S x_{2 n+1}, S S x_{2 n+1}, A S x_{2 n+1}}(k / d), G_{T_{2 n+2}, T x_{2 n+2}, B x_{2 n+2}}(k / e),\right.
\end{aligned}
$$

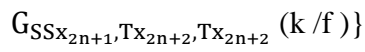

$$
\begin{aligned}
& \leq \min \left\{G_{S S x_{2 n+1}, S S x_{2 n+1}, A S x_{2 n+1}}(k / \beta), G_{T_{2 n+2}, T_{2 n+2}, B x_{2 n+2}}(k / \beta),\right. \\
& \mathrm{G}_{\left.\mathrm{SSx}_{2 \mathrm{n}+1}, \mathrm{Tx}_{2 \mathrm{n}+2}, \operatorname{Tx}_{2 \mathrm{n}+2}(\mathrm{k} / \beta)\right\}}
\end{aligned}
$$

Where $\beta=\min \{d, e, f\}$

Letting $\mathrm{n} \rightarrow \infty$, we have

$\mathrm{G}_{\mathrm{Sz}, \mathrm{z}, \mathrm{z}}(\mathrm{k}) \leq \min \left\{\mathrm{G}_{\mathrm{Sz}, \mathrm{Sz}, \mathrm{Sz}}(\mathrm{k} / \beta), \mathrm{G}_{\mathrm{z}, \mathrm{z}, \mathrm{z}}(\mathrm{k} / \beta), \mathrm{G}_{\mathrm{Sz}, \mathrm{z}, \mathrm{z}}(\mathrm{k} / \beta)\right\}$

Thus,

$\mathrm{G}_{\mathrm{Sz}, \mathrm{z}, \mathrm{z}}(\mathrm{k}) \leq \min \left\{\mathrm{G}_{\mathrm{Sz}, \mathrm{z}, \mathrm{z}}(\mathrm{k} / \beta)\right\} \Rightarrow \mathrm{G}_{\mathrm{Sz}, \mathrm{z}, \mathrm{z}}(\mathrm{k}) \leq \mathrm{G}_{\mathrm{Sz}, \mathrm{z}, \mathrm{z}}(\mathrm{k} / \beta)$

Since $\beta>1$, therefore $1 / \beta \in(0,1)$.

Using lemma (2.3), $\mathrm{Sz}=\mathrm{z}$.

Step 2. By putting $\mathrm{x}=\mathrm{z}, \mathrm{y}=\mathrm{x}_{2 \mathrm{n}+2}$ in (iv), we have

$$
\begin{aligned}
& \mathrm{G}_{\mathrm{Az}, \mathrm{Bx}_{2 \mathrm{n}+2}, \mathrm{Bx}_{2 \mathrm{n}+2}}(\mathrm{k}) \leq \min \left\{\mathrm{G}_{\mathrm{Sz}, \mathrm{Sz}, \mathrm{Az}}(\mathrm{k} / \mathrm{d}), \mathrm{G}_{\mathrm{Tx}_{2 \mathrm{n}+2}, \mathrm{Tx}_{2 \mathrm{n}+2}, \mathrm{Bx}_{2 \mathrm{n}+2}}(\mathrm{k} / \mathrm{e}\right. \\
& ), \mathrm{G}_{\mathrm{Sz}, \mathrm{Tx}_{2 \mathrm{n}+2}, \mathrm{Tx}_{2 \mathrm{n}+2}}(\mathrm{k} / \mathrm{f})\right\} \\
& \leq \min \left\{\mathrm{G}_{\mathrm{Sz}, \mathrm{Sz}, \mathrm{Az}}(\mathrm{k} / \beta), \mathrm{G}_{\mathrm{Tx}_{2 \mathrm{n}+2}, \mathrm{Tx}_{2 \mathrm{n}+2}, \mathrm{Bx}_{2 \mathrm{n}+2}}(\mathrm{k} / \beta),\right.
\end{aligned}
$$

$\left.\mathrm{G}_{\mathrm{Sz}, \mathrm{Tx}_{2 \mathrm{n}+2}, \mathrm{Tx}_{2 \mathrm{n}+2}}(\mathrm{k} / \beta)\right\}$

Where $\beta=\min \{d, e, f\}$

Letting $\mathrm{n} \rightarrow \infty$, we have

$$
\begin{aligned}
G_{A z, z, z}(k) & \leq \min \left\{G_{S z, S z, A z}(k /), G_{z, z, z}(k / \beta), G_{S z, z, z}(k / \beta)\right\}, \\
& \leq \min \left\{G_{z, z, A z}(k / \beta), G_{z, z, z}(k / \beta), G_{z, z, z}(k / \beta)\right\}, \\
& \leq G_{z, z, A z}(k / \beta)
\end{aligned}
$$

i.e. $G_{A z, z, z}(k) \leq G_{A z, z, z}(k / \beta)$, as $\beta>1$.

Hence, $\mathrm{Az}=\mathrm{z}$. Thus, $\mathrm{Sz}=\mathrm{z}=\mathrm{Az}$.

As $\mathrm{T}(\mathrm{X}) \subseteq \mathrm{A}(\mathrm{X})$, then there exists $\mathrm{w} \in \mathrm{X}$ such that $\mathrm{Tw}=\mathrm{Az}$ for some $\mathrm{z} \in \mathrm{X}$.

Therefore, $\mathrm{z}=\mathrm{Az}=\mathrm{Sz}=\mathrm{Tw}$.

Step 3. By putting $x=x_{2 n+1}, y=w$ in (iv), we have

$\mathrm{G}_{\mathrm{Ax}_{2 \mathrm{n}+1}, \mathrm{Bw}, \mathrm{Bw}}(\mathrm{k}) \leq \min \left\{\mathrm{G}_{\mathrm{Sx}_{2 \mathrm{n}+1}, \mathrm{Sx}_{2 \mathrm{n}+1}, \mathrm{Ax}_{2 \mathrm{n}+1}}(\mathrm{k} / \mathrm{d}), \mathrm{G}_{\mathrm{Tw}, \mathrm{Tw}, \mathrm{Bw}}(\mathrm{k} / \mathrm{e}\right.$

), $\left.\mathrm{G}_{\mathrm{Sx}_{2 \mathrm{n}+1}, \mathrm{Tw}, \mathrm{Tw}}(\mathrm{k} / \mathrm{f})\right\}$

$\leq \min \left\{\mathrm{G}_{\mathrm{Sx}_{2 \mathrm{n}+1}, \mathrm{Sx}_{2 \mathrm{n}+1}, \mathrm{Ax}_{2 \mathrm{n}+1}}(\mathrm{k} / \beta), \mathrm{G}_{\mathrm{Tw}, \mathrm{Tw}, \mathrm{Bw}}(\mathrm{k} / \beta)\right.$,

$\left.\mathrm{G}_{\mathrm{Sx}_{2 \mathrm{n}+1}, \mathrm{Tw}, \mathrm{Tw}}(\mathrm{k} / \beta)\right\}$

Letting $\mathrm{n} \rightarrow \infty$, we have

$\mathrm{G}_{\mathrm{z}, \mathrm{Bw}, \mathrm{Bw}}(\mathrm{k}) \leq \min \left\{\mathrm{G}_{\mathrm{z}, \mathrm{Z}, \mathrm{z}}(\mathrm{k} / \mathrm{d}), \mathrm{G}_{\mathrm{Tw}, \mathrm{Tw}, \mathrm{Bw}}(\mathrm{k} / \mathrm{e}), \mathrm{G}_{\mathrm{z}, \mathrm{Tw}, \mathrm{Tw}}(\mathrm{k} / \mathrm{f})\right\}$

$$
\begin{aligned}
& \leq \min \left\{G_{\mathrm{z}, \mathrm{z}, \mathrm{z}}(\mathrm{k} / \beta), \mathrm{G}_{\mathrm{z}, \mathrm{z}, \mathrm{Bw}}(\mathrm{k} / \beta), \mathrm{G}_{\mathrm{z}, \mathrm{z}, \mathrm{z}}(\mathrm{k} / \beta)\right\} \\
& \leq \mathrm{G}_{\mathrm{z}, \mathrm{z}, \mathrm{Bw}}(\mathrm{k} / \beta) \\
& \leq \mathrm{G}_{\mathrm{z}, \mathrm{Bw}, \mathrm{Bw}}(\mathrm{k} / \beta)
\end{aligned}
$$

i.e. $G_{z, B w, B w}(k) \leq G_{z, B w, B w}(k / \beta)$, as $\beta>1$.

As $(\mathrm{B}, \mathrm{T})$ is weak compatible, we have $\mathrm{TBw}=\mathrm{BTw} \Rightarrow \mathrm{Bz}=\mathrm{Tz}$.

Step 4. By putting $x=z, y=z$ in (iv), we have

$\mathrm{G}_{\mathrm{Az}, \mathrm{Bz}, \mathrm{Bz}}(\mathrm{k}) \leq \min \left\{\mathrm{G}_{\mathrm{Sz}, \mathrm{Sz}, \mathrm{Az}}(\mathrm{k} / \mathrm{d}), \mathrm{G}_{\mathrm{Tz}, \mathrm{Tz}, \mathrm{Bz}}(\mathrm{k} / \mathrm{e}), \mathrm{G}_{\mathrm{Sz}, \mathrm{Tz}, \mathrm{Tz}}(\mathrm{k} / \mathrm{f}\right.$ )\}

$\leq \min \left\{\mathrm{G}_{\mathrm{Sz}, \mathrm{Sz}, \mathrm{Az}}(\mathrm{k} / \beta), \mathrm{G}_{\mathrm{Tz}, \mathrm{Tz}, \mathrm{Bz}}(\mathrm{k} / \beta), \mathrm{G}_{\mathrm{Sz}, \mathrm{Tz}, \mathrm{Tz}}(\mathrm{k} / \beta)\right\}$

$\leq \min \left\{G_{z, z, z}(k / \beta), G_{z, z, B z}(k / \beta), G_{z, B z, B z}(k / \beta)\right\}$

$\leq \min \left\{G_{z, z, z}(k / \beta), G_{z, B z, B z}(k / \beta), G_{z, B z, B z}(k / \beta)\right\}$

$\mathrm{G}_{\mathrm{Az}, \mathrm{Bz}, \mathrm{Bz}}(\mathrm{k})=\mathrm{G}_{\mathrm{z}, \mathrm{Bz}, \mathrm{Bz}}(\mathrm{k}) \leq \mathrm{G}_{\mathrm{z}, \mathrm{Bz}, \mathrm{Bz}}(\mathrm{k} / \beta)$, Thus $\mathrm{z}=\mathrm{Bz}$.

Therefore $\mathrm{z}=\mathrm{Bz}=\mathrm{Tz}$. Hence, $\mathrm{z}=\mathrm{Az}=\mathrm{Sz}=\mathrm{Bz}=\mathrm{Tz}$.

Therefore, $\mathrm{z}$ is a common fixed point of $\mathrm{A}, \mathrm{B}, \mathrm{S}$ and $\mathrm{T}$.

Case II. A is continuous.

In this case, we have $\mathrm{ASx}_{2 \mathrm{n}+1} \rightarrow \mathrm{Az}$ and $\mathrm{A}^{2} \mathrm{x}_{2 \mathrm{n}+1} \rightarrow \mathrm{Az}$. and the semi-compatibility of (A, S) gives $\mathrm{ASx}_{2 \mathrm{n}+1} \rightarrow \mathrm{Sz}$. $\mathrm{By}$ uniqueness of limit in Menger space, we get $\mathrm{Az}=\mathrm{Sz}$.

Step 5. By putting $x=z, y=x_{2 n+1}$ in (IV), we have

$\mathrm{G}_{\mathrm{Az}, \mathrm{Bx}_{2 \mathrm{n}+1}, \mathrm{Bx}_{2 \mathrm{n}+1}}(\mathrm{k}) \leq \min \left\{\mathrm{G}_{\mathrm{Sz}, \mathrm{Sz}, \mathrm{Az}}(\mathrm{k} / \mathrm{d})\right.$,

$\left.\mathrm{G}_{\mathrm{Tx}_{2 \mathrm{n}+1}, \mathrm{Tx}_{2 \mathrm{n}+1}, \mathrm{Bx}_{2 \mathrm{n}+1}}(\mathrm{k} / \mathrm{e}), \mathrm{G}_{\mathrm{Sz}, \mathrm{Tx}_{2 \mathrm{n}+1}, \mathrm{Tx}_{2 \mathrm{n}+1}}(\mathrm{k} / \mathrm{f})\right\}$ 
$\leq \min \left\{\mathrm{G}_{\mathrm{Sz}, \mathrm{Sz}, \mathrm{Az}}(\mathrm{k} / \beta), \mathrm{G}_{\mathrm{Tx}_{2 \mathrm{n}+1}, \mathrm{Tx}_{2 \mathrm{n}+1}, \mathrm{Bx}_{2 \mathrm{n}+1}}(\mathrm{k} / \beta)\right.$,

$\left.\mathrm{G}_{\mathrm{Sz}, \mathrm{Tx}_{2 \mathrm{n}+1}, \mathrm{Tx}_{2 \mathrm{n}+1}}(\mathrm{k} / \beta)\right\}$

As $\beta=\min \{d, e, f\}$.

Letting $\mathrm{n} \rightarrow \infty$, we have

$\mathrm{G}_{\mathrm{Az}, \mathrm{z}, \mathrm{z}}(\mathrm{k}) \leq \min \left\{\mathrm{G}_{\mathrm{Sz}, \mathrm{Sz}, \mathrm{Az}}(\mathrm{k} / \beta), \mathrm{G}_{\mathrm{z}, \mathrm{z}, \mathrm{z}}(\mathrm{k} / \beta), \mathrm{G}_{\mathrm{Sz}, \mathrm{z}, \mathrm{z}}(\mathrm{k} / \beta)\right\}$

$\leq \min \left\{G_{\mathrm{Az}, \mathrm{Az}, \mathrm{Az}}(\mathrm{k} / \beta), \mathrm{G}_{\mathrm{z}, \mathrm{z}, \mathrm{z}}(\mathrm{k} / \beta), \mathrm{G}_{\mathrm{Az}, \mathrm{z}, \mathrm{z}}(\mathrm{k} / \beta)\right\}$

i.e. $G_{A z, z, z}(k) \leq G_{A z, z, z}(k / \beta)$, Thus, $z=A z$.

Hence, $\mathrm{z}=\mathrm{Az}=\mathrm{Sz}=\mathrm{Bz}=\mathrm{Tz}$, that is, $\mathrm{z}$ is a common fixed point of $\mathrm{A}, \mathrm{B}, \mathrm{S}$ and $\mathrm{T}$.

Now, let $\mathrm{u}$ be another common fixed point of $\mathrm{A}, \mathrm{B}, \mathrm{S}$ and $\mathrm{T}$, then $\mathrm{u}=\mathrm{Au}=\mathrm{Su}=\mathrm{Bu}=\mathrm{Tu}$.

So, by putting $\mathrm{x}=\mathrm{u}$ and $\mathrm{y}=\mathrm{z}$ in (iv), we have

$\mathrm{G}_{\mathrm{Au}, \mathrm{Bz}, \mathrm{Bz}}(\mathrm{k}) \leq \min \left\{\mathrm{G}_{\mathrm{Su}, \mathrm{Su}, \mathrm{Au}}(\mathrm{k} / \mathrm{d}), \mathrm{G}_{\mathrm{Tz}, \mathrm{Tz}, \mathrm{Bz}}(\mathrm{k} / \mathrm{e}), \mathrm{G}_{\mathrm{Su}, \mathrm{Tz}, \mathrm{Tz}}(\mathrm{k} / \mathrm{f}\right.$ )$\}$

$\leq \min \left\{G_{\mathrm{Su}, \mathrm{Su}, \mathrm{Au}}(\mathrm{k} / \beta), \mathrm{G}_{\mathrm{Tz}, \mathrm{Tz}, \mathrm{Bz}}(\mathrm{k} / \beta), \mathrm{G}_{\mathrm{Su}, \mathrm{Tz}, \mathrm{Tz}}(\mathrm{k} / \beta)\right\}$

$\Rightarrow \mathrm{G}_{\mathrm{u}, \mathrm{z}, \mathrm{z}}(\mathrm{k}) \leq \min \left\{\mathrm{G}_{\mathrm{u}, \mathrm{u}, \mathrm{u}}(\mathrm{k} / \beta), \mathrm{G}_{\mathrm{z}, \mathrm{z}, \mathrm{z}}(\mathrm{k} / \beta), \mathrm{G}_{\mathrm{u}, \mathrm{z}, \mathrm{z}}(\mathrm{k} / \beta)\right\} \leq$ $\mathrm{G}_{\mathrm{u}, \mathrm{z}, \mathrm{z}}(\mathrm{k} / \beta)$

Hence, $G_{u, z, z}(k) \leq G_{u, z, z}(k / \beta), k>0, \beta>1 . \Rightarrow u=z$.

Therefore, $\mathrm{z}$ is the unique common fixed point of $\mathrm{A}, \mathrm{B}, \mathrm{S}$ and $\mathrm{T}$.

Remark 2.7: Since every metric space is a Menger space, all results in Menger space, with some suitable modifications, can be applied to metric spaces, such as theorems 3.1, and 3.2 in [26].

\section{References}

[1] R. Vasuki, Fixed point and common fixed point theorems for expansive maps in Menger Spaces,

[2] Bull. Cal. Math. Soc., (1991), 83: pp.565- 570.

[3] R. Gujetiya and et al,Common Fixed Point Theorem for Expansive Maps in Menger Spaces through CompatibilityInter, Math. Forum, 5, 2010, no. 63, pp. $3147-3158$.

[4] D. Dolthinov, On Completeness in quasi-metric spaces, Journal of Topology and its Applications 30 (1988) , pp.127-148.

[5] S. Czerwik, Contraction mappings in b-metric spaces, Acta Math Univ. Ostrav. 1, (1993), pp. 5-11.

[6] A. Branciari, A fixed point theorem of Banach-Caccioppoli type on a class of generalized metric spaces, publ. Math. Debrcen, 57:1-2, (2000), pp.31-37.

[7] S. V. R. Naidu, Some fixed point theorems in metric spaces by altering distances Czechoslovak Mathematical Journal, Vol. 53,No.1, (2003), pp.205-212. http://dx.doi.org/10.1023/A:1022991929004

[8] L.G.Huang., X. Zhang, Cone metric spaces and fixed point theorems of contractive mappings, Journal of Mathematical Analysis and Applications 332(2007), pp.1467-1475. http://dx.doi.org/10.1016/j.jmaa.2005.03.087

[9] Z.Mastafa , B.Sims , Fixed Point Theorems for Contractive Mappings in Complete G-Metric Spaces, Fixed point Theory and Applications, Vol. (2009), pp.10

[10] S.K. Mohanta, and S. Mohanta, A Common fixed point theorem in G-metric spaces, CUBO A Mathematical Journal ,Vol.14, No 03, (2012), pp.85-101.

[11] M. Jleli and B. Samet, Remarks on G-metric spaces and fixed point theorems, Jleli and Samet Fixed Point Theory and Applications (2012), pp.1-7.

[12] M. Abbasa, A. R. Khanb, T.Nazira , Common fixed point of multivalued mappings in ordered generalized metric spaces, Filomat 26:5(2012), pp.1045-1053. http://dx.doi.org/10.2298/FIL1205045A

[13] W. Shatanawi, Some fixed point theorems in ordered G-metric spaces and Applications, Hindawi Publishing Corporation Abstract and Applied Analysis, Volume 2011, Article ID 126205, pp.1-11, http://dx.doi.org/10.1155/2011/126205.
[14] S.S. Abed, H. H. Luaibi, Two fixed point theorems in generalized metric spaces. International Journal of Advanced Statistics and Probability, 4 (1) (2016) 16-19. http://dx.doi.org/10.14419/ijasp.v4i1.5715.

[15] S.S.Abed, H. H. Luaibi, Fixed point theorems for condensing mappings in G-spaces, International Journal of Mathematical Sciences, ISSN: 2051-5995, Vol.35, Issue.1 (2015), pp. 1701- 1711.

[16] K.Menger , Statistical Metrics, Proc. Nat. Acad. Sci. U.S.A. 28 (1942), pp. 535-537. http://dx.doi.org/10.1073/pnas.28.12.535.

[17] S.S. Chag., Y.J.Lee, Y.Q.Chen S.M.Kang and J.S.Jung, Generized contraction mapping principle and differential equations in probabilistic metric spaces, Proc. Amer. Math. Soc.124 (1996), pp. 2367 2376. http://dx.doi.org/10.1090/S0002-9939-96-03289-3.

[18] V.M. Sehgal, Bharucha-Reid A. T., Fixed Points of Contraction Mappings on Probabilistic Metric Spaces, Math. Systems Theory, vol.6 (1972), pp. 97-102. http://dx.doi.org/10.1007/BF01706080.

[19] B.Schweizer, A. Sklar, Probabilistic Metric Space, North- Holland series in Probability and Applied math., North- Holland and pubishing co. Newyork, (1983)

[20] B.D. Pant, B. Fisher, S. Chauhan, Fixed Point Theorems in Probabilistic Metric Spaces Using Property (E.A), Mathematica Moravica ,Vol. 17-1(2013), pp. 11-24.

[21] S. Chander, Fixed Point Theorems for Occasionally Weakly Compatible Maps in Probabilistic Semi-Metric Spaces, Int. J. Math. Andl. vol. 3, (2009), pp.563 - 570.

[22] T.L.Hicks, Fixed Point Theory in Probabilistic Metric Spaces, Zbornik Radova, Prir.-

[23] M.Janfada ,A.R. Janfada , Z.Mollaee, Probabilistic G-Metric Space and Some Fixed Point Results, Journal Nonlinear Analysis and Appl. , ID: jnaa-00190,(2013) pp.2-10.

[24] S.S. Abed, H. H. Luaibi , Implicit fixed points in Menger G- metric spaces, International Journal of Advanced Scientific and Technical Research Issue 6 volume 1,(2016), pp.117-127.

[25] B.D.Pant, S. Chauhan, Common Fixed Point Theorems for Occasionally Weakly Compatible Mappings in Menger Probabilistic Quasi-Metric spaces, Adv. Nonlinear Var. Ineq.,14( 2), (2011), pp. 55-63.

[26] M. Kumar, Compatible Maps in G-Metric Spaces, Int. Journal of Math. Analysis, Vol. 6, No. 29 (2012), pp. 1415 - 1421.

[27] S. Chauhan, S. Kumar, Fixed point theorems in Menger spaces and applications to metric spaces, J. Appl. Math. \& Informatics Vol. 30, No. 5 - 6, (2012), pp. $729-740$.

[28] I. Golet, On Contractions in Probabilistic Metric Space, Radovi Mathematic, Vol.13, (2004), pp.87-92.

[29] V.M. Sehgal, Some Fixed Point Theorems in Functional Analysis and Probability, Wayne State Univ., Detroit, 1966, Ph. D. Dissertation

[30] N.Tahat, H.Aydi ,E. Karapinar,W.Shatanawi , Common Fixed Points For Single-valued and Multi-valued Maps Satisfying A Generalized Contraction in G-Metric Spaces, Tahat et al. Fixed Point Theory and Applications (2012), 2012:48 http://dx.doi.org/10.1186/1687-1812-2012-48. 